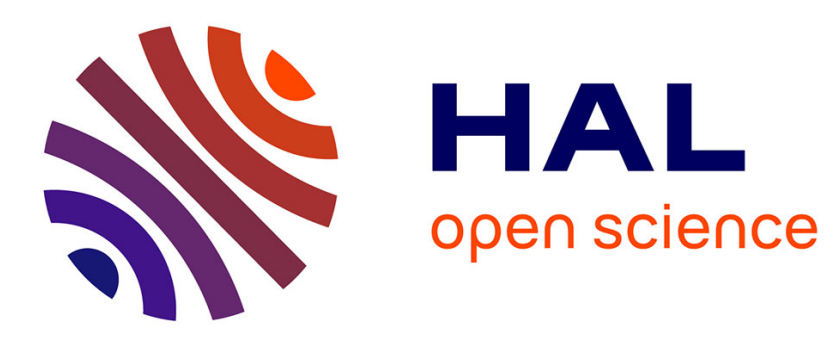

\title{
Structural sources identification through an inverse mid-high frequency energy method
}

\author{
Mohamed Chabchoub, Sébastien Besset, Mohamed Ichchou
}

\section{To cite this version:}

Mohamed Chabchoub, Sébastien Besset, Mohamed Ichchou. Structural sources identification through an inverse mid-high frequency energy method. Mechanical Systems and Signal Processing, 2011, 25

(8), pp.2948-2961. 10.1016/j.ymssp.2011.05.008 . hal-00625138

\section{HAL Id: hal-00625138 \\ https://hal.science/hal-00625138}

Submitted on 22 Sep 2011

HAL is a multi-disciplinary open access archive for the deposit and dissemination of scientific research documents, whether they are published or not. The documents may come from teaching and research institutions in France or abroad, or from public or private research centers.
L'archive ouverte pluridisciplinaire HAL, est destinée au dépôt et à la diffusion de documents scientifiques de niveau recherche, publiés ou non, émanant des établissements d'enseignement et de recherche français ou étrangers, des laboratoires publics ou privés. 


\title{
Structural sources identification through an inverse mid-high frequency energy method
}

\author{
M. A. Chabchoub, S. Besset and M. N. Ichchou \\ Laboratoire LTDS, Équipe D2S, UMR CNRS 5513 \\ École Centrale de Lyon, 36 av. Guy de Collongue \\ 69134 Ecully cedex, France
}

Mechanical Systems and Signal Processing, 25(8), 2948-2961, 2011

\begin{abstract}
This paper is primarily focused on the identification of structural forces, with the objective of localizing forces injected into structures in the mid-high frequency range. An energy method, called the Simplified Energy Method (MES), has already been introduced for the purpose of predicting an energy density distribution for structural acoustic problems in the mid-high frequency range. The present paper proposes using this same energy method to solve inverse structural problems. More specifically, the injected forces are to be estimated and localized through knowledge of a set of energy densities within the structure. The 2D formulation of this inverse approach, known as Inverse MES (or IMES), is first expressed. Both the boundary and internal sources can then be detected by applying the proposed formulation. Numerical test results are processed using a 2D Kirchhoff plate, and a number of conclusions are also drawn regarding IMES capabilities. Moreover, this paper offers a numerical comparison with another energy-based method.
\end{abstract}

keyword: Energy flow; MES; Forces identification; Forces localization; Injected power density; Energy density; Intensity

\section{Introduction}

The identification of force features that serve to excite structural components is a key concern in the field of structural engineering. The localization and quantification of input loads is of considerable interest; frequently, the direct measurement of sources proves quite complicated to perform. Indirect means for defining excitation often provides an effective alternative. A tremendous amount of work has been performed and published regarding this issue [1, 2, 3, 4, 5, 6, 7, 8, 9, 10, 11]. The problem being considered herein may also have applications in structural health monitoring. Damage, whose features often remain unknown, leads to changes in the vibration energy distribution of structural members. Both the magnitude and position detection of damage thus become key parameters. 
In general, two approaches are available in the literature relative to input force identification aspects. The first has more or less been inspired by identification approaches for the black box-like behavior of dynamic systems, coupled with the introduction of estimation theories. As an example, in references [1, 2], the author uses an online recursive inverse method in order to estimate the input forces of beam and plate structures. The inverse method has been based on the Kalman filter plus a recursive least-squares algorithm that includes just a small number of measurement responses within the structure. Several types of input forces have been successfully identified. This method performs effectively in estimating the input forces of beam structural systems from noisy measurements. It has been shown [3] that a Kalman filter method may be used to estimate a timewise variation rod force. The second approach consists of defining the input force that allows the measured set of inputs to verify the equilibrium equations given in either continuous or discretized form. For example, a number of authors have developed a pertinent strategy, called the RIFF method, for identifying vibration sources [4, 5, 6]. The underlying principle entails both the discretization of motion equations via a finite difference scheme and the computation of force distribution from a set of displacements measured on the structure. Regularization is then achieved through a spatial window and a wave number filter. Feasibility studies were conducted for beam and plate elements, and an application to cylindrical shells has been proposed [6]. Recently, this approach was coupled to near-field acoustic holography in order to make a successful identification from acoustic measurements [5]. Perotin and Granger offered another approach, whereby a modal model of a structure along with a spatial orthonormal decomposition of the excitation field are used in order to derive the force identification technique [7]. The problem associated with the vibration input force localization has also been addressed in the structural intensity community; moreover, instead of applying an analytical model [4] or modal model [7] in the inversion process, discretized finite element $(\mathrm{FE})$ or boundary element $(\mathrm{BE})$ can be introduced into the identification [10, 11]. Noiseux [8, 9] wound up using intensity vectors to indicate the energy flow and source localization.

It should be noted however that most of what has been proposed in the literature focuses on a limited frequency band, more precisely the so-called low-frequency region where modal overlap is weak and where resonant behavior of the structure still dominates. As frequency increases, existing methods lose their advantage. The first such drawback pertains to the robustness of strategies offered relative to noise and uncertainties. It is expected that approaches based on the spatial discretization of data will be very sensitive to discretization errors over short wavelengths. A costly and time-consuming treatment is thus expected in order to deal with high-frequency band features. Moreover, identification strategies that use finite element, boundary element or simplified analytical modeling of the targeted structure are naturally confronted with intrinsic limitations when modeling in the mid-high frequency regime. The need is therefore expressed to establish an identification tool that takes into account both medium and high-frequency particularities. The use of an energy-based method, which seeks to produce a robust estimation of the average vibration level, is the approach adopted in this paper to treat the problem.

Energy methods are often used as alternatives in the medium and high frequency ranges whenever numerical methods like finite element or boundary equation-based 
formulations encounter significant limitations. Among such energy methods, the most widespread remains the Statistical Energy Analysis (SEA) [12], which yields the mechanical energy of complex built-up structures. Many energy methods have been based on SEA, in an attempt to enhance the Statistical Energy Analysis robustness and predictability. Let's cite the work of Nefske and Sung [13], who derived a local energy formalism. Many authors have improved on this model [14, 15], leading to the Simplified Energy Method (MES), which has been applied to various domains, including beams, plates and acoustic cavities [15, 20] as a "direct" prediction tool.

In this paper, the primary concern revolves around the use of MES in predicting energy sources [16]. This issue necessitates inverting the direct formulation. The paper will be structured as follows. To ensure clarity, the direct MES formulation will be reviewed in Section II, before providing the inverse MES formulation. A twodimensional case will then be considered and the algebraic matrix format of the inversion problem offered in Section III. In order to validate the proposed method, numerical simulations will be run in Section IV. A step-by-step strategy will be employed during the validation process. An initial step introduces direct estimated energies into the inversion sequence, while a second attempt simulates the input energy using a finite element code. A 2D plate will be studied, in addition to identifying surface sources. Ultimately, a comparison of the inverse MES results with an inverse SEA formulation will serve to complete the numerical experiments.

\section{Overview of the direct energy flow method MES}

\subsection{Assumptions}

The Simplified Energy Method (MES) is based on a description of two local energy quantities. The first is the total energy density $W$ defined as the sum of the potential energy density and the kinetic energy density. The second energy variable $\vec{I}$ is the energy flow. The energy balance can then be written as follows:

$$
\vec{\nabla} \cdot \vec{I}+\pi_{\text {diss }}=\pi_{\text {inp }}
$$

where $\vec{\nabla}$ is the gradient operator, $\pi_{\text {diss }}$ is the dissipated power density and $\pi_{i n p}$ the input power density. MES adopts the same damping model as SEA, as previously discussed in the literature [12], which can be expressed as:

$$
\pi_{\mathrm{diss}}=\eta \omega W
$$

where $\eta$ is the loss factor and $\omega$ the circular frequency. The propagating waves considered in this MES formulation are made of partial energy quantities corresponding to both direct and reverberated fields. Since the considered fields are quadratic variables, the superposition principle can thus be applied:

$$
£=\sum_{i} £_{i}
$$


where $\left(£, £_{i}\right)$ are quadratic variables corresponding to either global energy or a partial energy associated with wave fields $\left(W, W_{i}\right)$ or $\left(\vec{I}, \vec{I}_{i}\right)$. In the following, an intrinsic energy law will be introduced, and this often enables defining the wave velocity $c$ :

$$
\vec{I}^{i}=c \cdot W^{i} \vec{n}
$$

This expression simply defines the energy velocity, which in some cases is the same as the group velocity, as the ratio of energy flow to convected energy density. This expression was demonstrated in a number of textbooks [21] and applies to any kind of linear elastic propagating wave.

\subsection{Direct formulation}

In the following, the derivation of the MES formulation is offered by considering a $n$ Dimensional $(n \mathrm{D})$ space. The following equations are valid for $n=2$ or $n=3$ in the general case. In the next sections, we will consider the $n=2$ case, whereas the $n=3$ case will be treated in a subsequent article. In considering a symmetrical wave field and Equations (4) and (1), the MES approach [17] leads to the following relation:

$$
\frac{1}{r^{n-1}} \frac{\partial}{\partial r}\left(r^{n-1} \vec{I}\right)+\eta \omega W \vec{n}=0
$$

which can also be written using Equation (4) as:

$$
-c^{2} \frac{1}{r^{n-1}} \frac{\partial}{\partial r}\left(r^{n-1} W \vec{n}\right)=\eta \omega \vec{I}
$$

Moreover, this equation can now be rewritten by considering just the $W$ field, thus providing a local energy equation for symmetrical wave energies, i.e.:

$$
\frac{1}{r^{n-1}} \frac{\partial}{\partial r}\left(r^{n-1} W\right)+\frac{\eta \omega}{c} W=0
$$

Energy variables are defined as the superposition of a direct field and a reverberated field, both of which can be summed to obtain the total field, as explained in Equation (3). A representation of these fields is given in Figure (1), where only the intensity field has been shown. $\vec{I}^{\text {dir }}$ is the direct field, while $\vec{I}^{\text {ev }}$ is the reverberated field.

In Figure 1 in $\Phi^{\text {inp }}$ is the input power, $\Omega$ is the system surface and $\partial \Omega$ is the system boundaries. The elementary solutions in terms of energy density and active intensity are denoted $G$ and $\vec{H}$, respectively.

$$
G(S, M)=\frac{1}{S M} e^{-\frac{\eta \omega}{c} S M} \quad \vec{H}(S, M)=\frac{c}{S M} e^{-\frac{\eta \omega}{c} S M} \vec{u}_{S M}
$$

where $S M=\|S \vec{M}\|$. The kernels are determined at each point $\mathrm{M}$ of the considered space and created by a source located in S. By implementing a superposition principle, the energy solution can then be expressed using the contribution of the primary source $\rho$ (direct field) and fictitious sources $\sigma$. The fictitious sources are unknown 


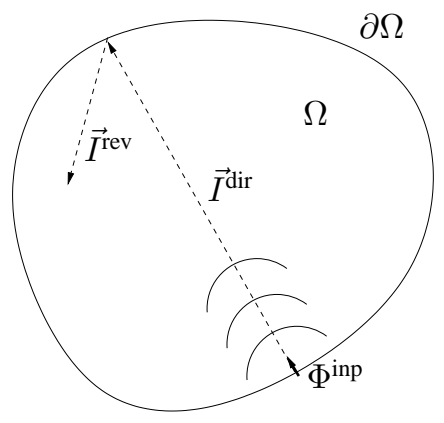

Figure 1: Considered system

energy parameters representing the contribution of the reverberant field. More specifically, from [20]:

$$
W(M)=\int_{\Omega} \rho(S) G(S, M) d S+\int_{\partial \Omega} \sigma(P) \vec{u}_{S M} \vec{n}_{P} G(P, M) d P
$$

The active energy flow can be described by a similar expression:

$$
I(M)=\int_{\Omega} \rho(S) H(S, M) d S+\int_{\partial \Omega} \sigma(P) \vec{u}_{S M} \vec{n}_{P} H(P, M) d P
$$

where $\rho$ corresponds to internal sources in the considered domain (in this case, a two dimensional) and $\sigma$ corresponds to sources at the boundaries of the two dimensional space.

\section{The inverse energy flow method IMES formulation}

This section will focus on introducing a discretized format of the energy integral equation, beginning with the definition of $T$ as the $(N, N)$ geometric interaction matrix between $N$ two-dimensional edge elements. This operator is given below in reference to Figure (2), with $P$ and $P^{\prime}$ corresponding to $P_{i}$ and $P_{j}$ (point $S$ only concerns equation 12

$$
T_{i j}=\int_{\partial \Omega} \int_{\partial \Omega} \frac{1}{2} \frac{1}{P_{i} P_{j}} e^{-\frac{\eta \omega}{c} P_{i} P_{j}} \vec{u}_{P_{i} P_{j}} \vec{n}_{P_{i}} d P_{i} d P_{j}
$$

In reference to this same Figure (2), another $(N, k)$ matrix is defined and called herein $Q$. This operator represents the geometric interaction taking place between $N$ boundary elements and $k$ sources. Figure (2) was drawn with $k$ set equal to 1 . When considering the inverse problem, matrices are built assuming $k$ sources, which will comprise the unknowns of this problem. For example, setting $k=m$ will lead to a square problem to be solved. Hence, even if it were known that just one source had to be found, the matrices would still be built with $k>1$, where $k$ sources were located on 


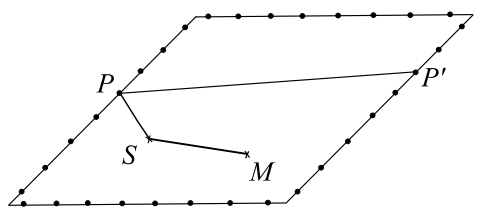

Figure 2: element/element interaction

the plate. The identified point should be the nearest to the source, and the other $(k-1)$ sources should be identified close to 0 . Matrix $Q$ is thus expressed as follows:

$$
Q_{i j}=\int_{\partial \Omega} \frac{1}{2} \frac{1}{S_{j} P_{i}} e^{-\frac{\eta \omega}{c} S_{j} P_{i}} \vec{u}_{S_{j} P_{i}} \vec{n}_{P_{i}} d P_{i}
$$

Two matrices, labeled $Y^{W}$ and $Y^{I}$ (with dimension $(k, m)$ where $m$ denotes the number of measurements) can then be defined in order to represent a geometric interaction between sources and measurement points. These matrices are determined respectively for the energy density $W$ and the energy flow $I$ such that:

$$
\begin{gathered}
Y_{i j}^{W}=\int_{\partial \Omega} \frac{1}{S_{j} P_{i}} e^{-\frac{\eta \omega}{c} S_{j} P_{i}} d P_{i} \\
Y_{i j}^{I}=\frac{1}{c} \int_{\partial \Omega} \frac{1}{S_{j} P_{i}} e^{-\frac{\eta \omega}{c} S_{j} P_{i}} \vec{u}_{S_{j} P_{i}} d P_{i}
\end{gathered}
$$

Ultimately, $A$ and $B$ can be defined as follows (where $I d$ being the identity matrix and $\rho$ the injected power vector):

$$
\begin{gathered}
A=I d-T \\
B=Q \rho
\end{gathered}
$$

The power vector $\sigma$ can thus be readily expressed as a function of the injected power vector $\rho$ as:

$$
\sigma=A^{-1} B=A^{-1} Q \rho
$$

By combining Equations (9), (10) and (17), the energy density vector $W$ and energy flow vector $I$ can be obtained from:

$$
\begin{gathered}
W=S^{W} \rho=R^{W} \rho+Y^{W} \sigma \\
I=S^{I} \rho=R^{I} \rho+Y^{I} \sigma
\end{gathered}
$$

where $R^{W}$ and $R^{I}$ are defined as follows:

$$
\begin{gathered}
R_{i j}^{W}=\frac{1}{S_{i} M_{j}} e^{-\frac{-\eta \omega}{c} S_{i} M_{j}} \\
R_{i j}^{I}=\frac{1}{S_{i} M_{j}} e^{-\frac{-\eta \omega}{c} S_{i} M_{j}} \vec{u}_{S_{i} M_{j}}
\end{gathered}
$$


$S^{W}$ and $S^{I}$ are then given by:

$$
\begin{gathered}
S^{W}=R^{W}+Y^{W} A^{-1} Q \\
S^{I}=R^{I}+Y^{I} A^{-1} Q
\end{gathered}
$$

Lastly, both the $W$ and $\vec{I}$ fields can be expressed thanks to a linear operator denoted $\mathcal{F}$ as:

$$
(W, \vec{I})=\mathcal{F}(\rho)
$$

The IMES formulation seeks to invert the operator $\mathcal{F}$ proposed in Equation (24). Sources are to be detected by means of measurements conducted on the structure. Since the number of measurements is not equal to the number of sources, this problem is not directly invertible, which prompts us to seek an operator $\mathcal{G}$ that yields $\sigma$ and $\rho$ as a function of $W$ and $\vec{I}$.

$$
\rho=\mathcal{G}(W, \vec{I})
$$

Now, let's introduce the quantity $R(\mathcal{X})$ defined as follows:

$$
R(\mathcal{X})=\left\|(W, \vec{I})-\mathcal{F}_{O} \mathcal{X}(W, \vec{I})\right\|
$$

where the operator $o$ is defined as $f \circ g(x)=f(g(x))$. We now need to minimize the quantity $R(\mathcal{X})$ over the operator $\mathcal{X}$. In the following discussion, the IMES will be applied to a $2 \mathrm{D}$ plate. Consequently, $\Omega$ corresponds to the plate surface, whereas $\partial \Omega$ corresponds to the boundaries. In this case, only surface sources are being treated. Since the access to structural intensities is a difficult task to perform experimentally, the following simulations have been run by only considering energy density $W$ measurements at certain locations of the structure. As a result, we will replace $S^{W}$ by $S$ for the sake of clarity. MES equation (9) can then be discretized to provide the following matrix formulation:

$$
\left\{\begin{array}{c}
W_{1} \\
W_{2} \\
\vdots \\
W_{m}
\end{array}\right\}=\left[\begin{array}{cccc}
S_{11} & S_{12} & \ldots & S_{1 k} \\
S_{21} & S_{22} & \ldots & S_{2 k} \\
\vdots & \vdots & \ddots & \vdots \\
S_{m 1} & S_{m 2} & \ldots & S_{m k}
\end{array}\right]\left\{\begin{array}{c}
\rho_{1} \\
\rho_{2} \\
\vdots \\
\rho_{k}
\end{array}\right\}
$$

where $m$ is the number of measurements and $k$ is the number of sources. To invert this formulation, according to Equation (26), a matrix $X$ must be found that minimizes the quantity $R(X)$ : 


$$
\begin{aligned}
R(X) & =\left\|\left\{\begin{array}{c}
W_{1} \\
W_{2} \\
\vdots \\
W_{m}
\end{array}\right\}-\left[\begin{array}{cccc}
S_{11} & S_{12} & \ldots & S_{1 k} \\
S_{21} & S_{22} & \ldots & S_{2 k} \\
\vdots & \vdots & \ddots & \vdots \\
S_{m 1} & S_{m 2} & \ldots & S_{m k}
\end{array}\right]\left[\begin{array}{cccc}
X_{11} & X_{12} & \ldots & X_{1 m} \\
X_{21} & X_{22} & \ldots & X_{2 m} \\
\vdots & \vdots & \ddots & \vdots \\
X_{k 1} & X_{k 2} & \ldots & X_{k m}
\end{array}\right]\left\{\begin{array}{c}
W_{1} \\
W_{2} \\
\vdots \\
W_{m}
\end{array}\right\}\right\| \\
& =\left\|\left(I_{m}-S X\right)\right\|\left\|\left\{\begin{array}{c}
W_{1} \\
W_{2} \\
\vdots \\
W_{m}
\end{array}\right\}\right\|
\end{aligned}
$$

Matrix $X$ is in fact chosen as a generalized inverse of matrix $\mathrm{S}$ :

$$
X=\left(S^{T} S\right)^{-1} S^{T}
$$

The inverse formulation can then be performed. From a set of input energy parameters, the inversion can be achieved in order to characterize input sources. Section 4 below will describe the efficiency and limitations of the proposed strategy through numerical experimentation.

\section{Numerical simulations and Results}

This section will perform a number of numerical tests on a plate in order to validate the formulation. The first test case considered will be very simple; it consists of implementing the inverse energy flow approach when using a set of energy predictions based on the direct energy flow method. This set-up constitutes an MES/IMES simulation. A more realistic test case will be examined afterwards. In this situation, the input variables are computed from a full finite element model. A comparison between the IMES formulation provided in this paper and an inverse SEA model will also be given. Lastly, the effect of spatial discretization applied to the input set of energy quantities on the inversion process will be studied.

\begin{tabular}{ccc}
\hline Case number & Direct method & Inverse method \\
\hline 1 & MES & IMES \\
2 & FEM & IMES \\
3 & FEM & Inverse SEA \\
\hline
\end{tabular}

Table 1: Numerical simulations

Table 1 summarizes the three test cases being considered herein. The corresponding methodology is presented in Figure 3

- The first one will allow verifying the potential for method inversion. If the matrix given in equation 30 were indeed invertible, then the results should be perfect. 
- The second case is intended to apply IMES in data originating from a finite element code. First of all, the structure is modeled using the finite element software COMSOL. Data are then extracted and treated through the IMES code, as explained in the previous section.

- The third case is similar to the second, yet an Inverse SEA software is used instead of the IMES code. The goal here is to compare the efficiency of IMES and Inverse SEA.

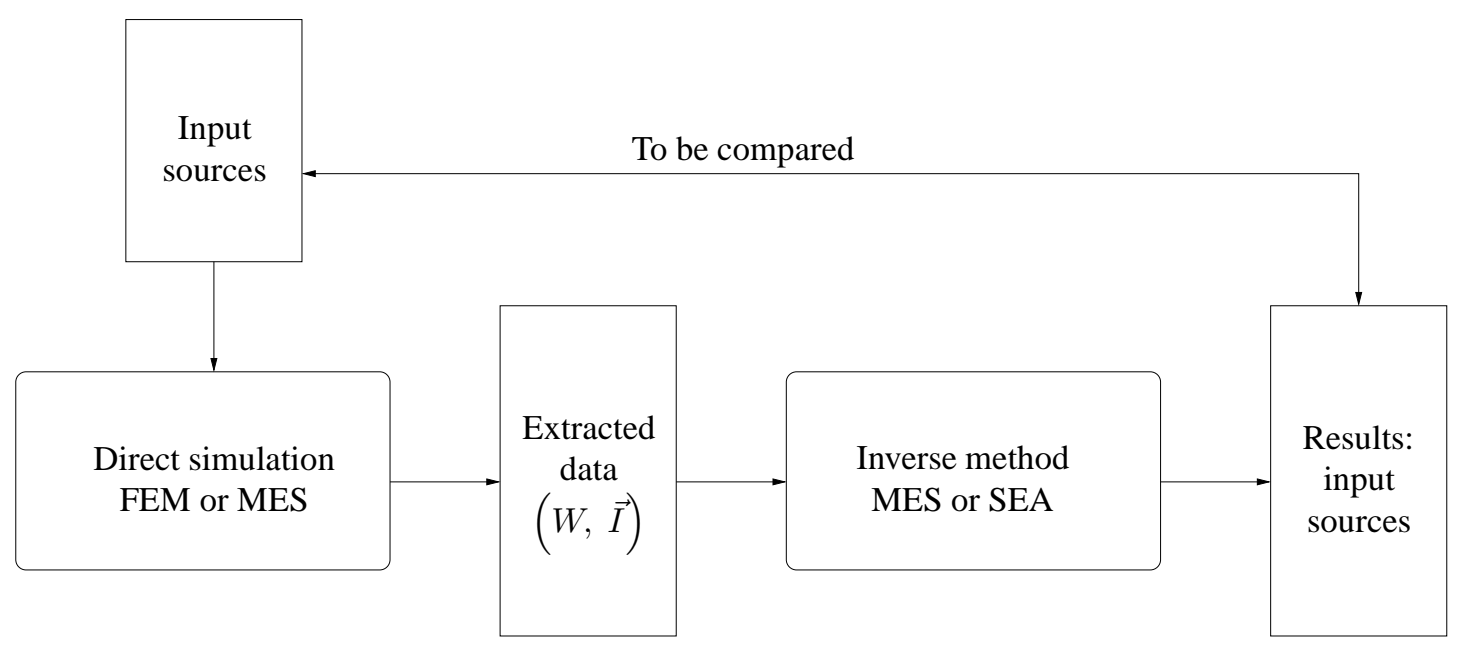

Figure 3: Numerical simulation methodology

\subsection{MES/IMES simulation}

An initial simulation, referred as the MES/IMES simulation, involved the processing of IMES calculations using energy densities $W$ derived from the direct MES formulation. The model under study in this case is a thin plate, whose characteristics are described in Table (2).

This plate is excited by an input power of $1 \mathrm{~W} / \mathrm{m}^{2}$. In assuming $N$ to be the number of edge plate elements, the IMES result found for $N=m=64$ is presented in Figure (4) (let's recall that $m$ denotes the number of measurements).

It appears that the source has been correctly identified in this simulation (for both localization and level). The inversion of $\left(S^{T} S\right)$ has thus been validated. To illustrate the effect of the number of measurements $m$, we repeated the MES/IMES simulation while decreasing $m$. The IMES results are shown in figure (5). Next, let's then introduce the factor $\xi=\frac{m}{N}$ to quantify method efficiency ( $\xi=1$ corresponds to a square matrix to be inverted).

It also appears that by increasing the number of measurements $m$ spread over the plate surface corresponding to $\xi=0.06, \xi=0.14$ and $\xi=0.5$ respectively, the 


\begin{tabular}{ccc}
\hline parameter & unit & value \\
\hline Length & $m$ & 1 \\
Width & $m$ & 1 \\
Height & $m$ & $2 e-3$ \\
Young's modulus of elasticity $E$ & $P a$ & $2.1 e 11$ \\
Poisson's ratio $\nu$ & & 0.3 \\
Solid density $\rho_{s}$ & $K g / m^{3}$ & 7800 \\
Loss factor & & $\eta=0.001$ \\
\hline
\end{tabular}

Table 2: plate caracteristics

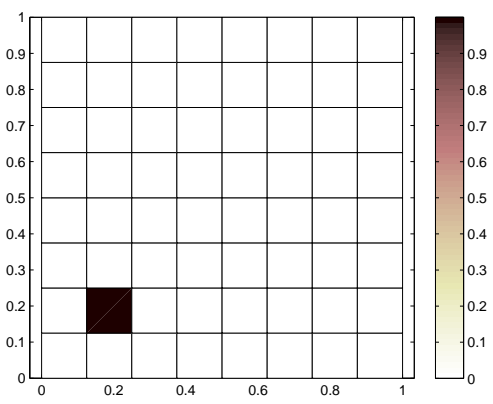

Figure 4: MES/IMES simulation for the plate

source is well localized and the error on the input power summarized in Table (3) has been lowered. Nevertheless, the source input power is equal to $0.7 \mathrm{~W} / \mathrm{m}^{2}$ for $\xi=0.06,0.75 \mathrm{~W} / \mathrm{m}^{2}$ for $\xi=0.14$ and $0.82 \mathrm{~W} / \mathrm{m}^{2}$ for $\xi=0.5$ whereas it should equal $1 \mathrm{~W} / \mathrm{m}^{2}$. The correct value of input power is obtained for higher values of $\xi$. The IMES tends to accurately localize the input structural force yet underestimates the amount of injected power.

\subsection{FEM/IMES simulation}

The IMES/MES subsection primarily allowed validating the $\left(S^{T} S\right)$ inversion. This subsection will consider the validation of an approach introducing FEM-simulated en-

\begin{tabular}{cc}
\hline$\xi$ & Error \\
\hline 0.06 & $30 \%$ \\
0.14 & $25 \%$ \\
0.5 & $18 \%$ \\
\hline
\end{tabular}

Table 3: Error on the input power 
ergies. The IMES calculations were in fact processed using energy densities $W$ stemming from a finite element software (i.e. Comsol in this study). An FEM model of the plate (whose characteristics are provided below) has been processed with $1 \mathrm{~W} / \mathrm{m}^{2}$ excitation and the same number of elements $N$. Given the input power $\rho$, the force $F$ applied to a point on the plate is recalculated by:

$$
\rho=\frac{1}{2} \frac{F^{2}}{8 \sqrt{D \rho_{s} h}}
$$

Where $D$ is the plate stiffness, defined by $D=\frac{E h^{3}}{12\left(1-\nu^{2}\right)}$. The FEM calculation is then processed on the plate, thus providing the energy density $W$ field used as an input for IMES algorithm. The FEM calculations are first spatially averaged and then frequency averaged. Octave bands are considered along with various center frequencies $f_{c}$. The values of $W$ output by the FEM calculation and performed around $f_{c}$, are input into the IMES algorithm. Results found for $\xi=0.06, \xi=0.14, \xi=0.5$ and $\xi=1$ are all presented in figure (6).

The influence of $f_{c}$ has also been assessed. Results found for $\xi=1$ and various values of $f_{c}$ are given in figure (7). This figure reveals an accurate source localization regardless of the $f_{c}$ value within the medium to high frequency range.

Figure (8) displays the evolution of a localization term $\operatorname{Pr}_{L}$ with respect to the center frequency. $P r_{L}$ is defined by the following equation:

$$
\operatorname{Pr}_{L}=\sqrt{\frac{1}{N} \frac{\sum_{i=1}^{N}\left(\rho_{i}-\rho_{i}^{\text {exact }}\right)^{2}}{\left(\rho_{i}^{\text {exact }}\right)^{2}}}
$$

where $\rho_{i}$ is the $i$ component of the input power vector and $\rho_{i}^{\text {exact }}$ is the $i$ component of the exact input power vector. It would appear that a good identification (both localization and level) is derived for the source with values of $f_{c}=950 \mathrm{~Hz}$ and higher. For lower-frequency bands, the proposed method will only be able to localize the sources.

\subsection{SEA / MES comparison}

The statistical Energy Analysis (SEA) is widely recognized as the original class of energy methods. SEA provides the mechanical energy of complex built-up structures. The goal of this subsection is to compare identification results obtained for FEM / SEA and FEM / IMES simulations. Towards this end, Figures (9) and (10) indicate the identification tendencies for various damping values when using FEM/IMES and FEM/SEA identification strategies, respectively.

In all cases, the source seems to be correctly localized, though the estimated value of the input power $\rho$ deviates from the expected value $\rho^{\text {exact }}$ with increasing $\eta$. To identify the localization sensitivity in figure 11 a)), we introduce the same localization term $\operatorname{Pr}_{L}$ given in Equation (32); moreover, representation of the quantification sensitivity in Figure (11 b)) has led us to define another quality term $\operatorname{Pr}_{Q}$ obtained from the following equation: 


$$
\operatorname{Pr}_{Q}=\sqrt{\frac{\left(\rho_{\text {source }}-\rho_{\text {source }}^{\text {exact }}\right)^{2}}{\left(\rho_{\text {source }}^{\text {exact }}\right)^{2}}}
$$

It appears that for both the localization and quantification of sources, the FEM/IMES results underestimates the FEM/SEA tendencies, which reflects that IMES that IMES results are more efficient than ISEA results in identifying sources for the studied plate. This finding would also suggest that the capacity of FEM/IMES localization remains more stable when varying $\eta$ than FEM/ISEA localization results, for which this precision term increases when increasing $\eta$. Moreover, from both the FEM/IMES and FEM/ISEA quantification trends, it can be remarked that good results are obtained with weak damping values.

\subsection{Effect of spatial discretization (sensors spread away from the source)}

In this subsection, the IMES validation will be considered in a realistic case, according to which sensors are removed from the source. The IMES simulations were performed using a set of uniformly-distributed sensors far from the source location. Results relative to these FEM/IMES simulation runs and sensors distribution in the plate for $m=55$ and $m=39$ are given in figure 12 .

The results obtained indicate that the source has been correctly localized, yet a discrepancy remains between the estimated input power $\rho$ is and the expected power $\rho^{\text {exact }}$ due to the lack of sensors close to the source. To analyze the localization sensitivity of this method with respect to parameter $\xi$ defined below, Figure (13) depicts the evolution in quality index $\operatorname{Pr}_{L}$ evolution. This figure provides a presentation of localization effectiveness.

It has thus been demonstrated that by using the IMES formulation, source localization can be achieved while limiting the number of sensors and distributing them far from the source.

\section{Conclusion}

The aim of this paper has been to investigate the effectiveness of the Simplified Energy Method (MES) in identifying sources from a set of given measured energies within a plate. The MES formulation and related assumptions were first detailed. The inverse formulation for identifying sources through data energy recovered from the plate was then provided. Afterwards, the MES could be validated using FEM / MES simulations by inputting numerical data from the FEM software. A comparison with SEA was also conducted and revealed that IMES is indeed more accurate. Lastly, a robustness study was performed, displaying the MES performance when sensors were removed from the source. Validation of this strategy for 3D acoustic cavities is now underway. In subsequent work, it will be worthwhile to test this method by considering two or more sources. From a theoretical perspective, the matrices and formulation proposed herein should be capable of treating this type of problem, yet numerical considerations 
complicate the identification step. Moreover, it would be beneficial to validate the method through experimental testing since the noise induced by "real" measurements should lead to findings regarding the inversion. From a numerical standpoint, it would be interesting to introduce a random noise on the input data in order to estimate the robustness of the inverse method proposed in this paper.

\section{References}

[1] Jui-Jung Liu, Chin-Kao Ma, I-Chung Kung and Dong-Cherng Lin, Input force estimation of a cantilever plate by using a system identification technique, Computer Methods in Applied Mechanics and Engineering, 190, Issues 11-12, Pages 1309-1322, 2000.

[2] C. -K. Ma, J. -M. Chang and D. -C. Lin, Input force estimation of beam structures by an inverse method, Journal of Sound and Vibration, 259, Issue 2, Pages 387407, 2003.

[3] C-C. Ji and C. Liang, A study on an estimation method for applied force on the rod, Computer Methods in Applied Mechanics and Engineering, 190, Issues 1112, Pages 1209-1220, 2000.

[4] C. Pezerat and J. L. Guyader, Identification of vibration sources, Applied Acoustics, 63, Pages 309-324, 2000.

[5] M. C. Djamaa, N. Ouelaa, C. Pezerat and J. L. Guyader, Reconstruction of a distributed force applied on a thin cylindrical shell by an inverse method and spatial filtering, Journal of Sound and Vibration, 301, Pages 560-575, 2007.

[6] C. Pezerat, Q. Leclére, N. Totaro and M. Pachebat, Identification of vibration excitations from acoustic measurements using near field acoustic holography and the force analysis technique, Journal of Sound and Vibration, 326, Pages 540-556, 2009.

[7] L. Perotin and S. Granger, An inverse method for the identification of a distributed random excitation acting on a vibrating structure: Part 2, flow induced vibration application, Mechanical Systems and Signal Processing, 13, Issue 1, Pages 67-81, 1999.

[8] D. U. Noiseux, Measurement of power flow in uniform beams and plates, Journal of Acoustical Society of America, 47, Pages 238-247, 1970.

[9] G. Pavic, Measurement of structure borne wave intenisty: Part I, formulation of the methods, Journal of Sound and Vibration, 49, Issue 2, Pages 221-230, 1976.

[10] M. A. Hamdi, H. Defosse and L. Gagliardini, Hybrid inverse boundary element method for the determination of the optimal spectral characteristics of complex radiating noise sources, Internoise, Dearborn, MI, USA, 2002. 
[11] M. R. Bai, Application of BEM-based acoustic holography to radiation analysis of sound sources with arbitrarily shaped geometries, Journal of Acoustical Soc. Am. 92, 533-549, 1992.

[12] R. H. Lyon, Statistical Energy Analysis of Dynamical Systems: Theory and Application, Cambridge, Massachusetts, MIT Press, 1975.

[13] D. J. Nefske and S.H. Sung, Power Flow Finite Element Analysis of Dynamic Systems: Basic Theory and Application to Beams, NCA Publication, 3, 1987.

[14] J. C. Wohlever and R. J. Bernhard, Mechanical Energy Flow Models of Rods and Beams, Journal of Sound and Vibration, 153, 1619, 1992.

[15] Y. Lase, M. N. Ichchou and L. Jezequel:, Energy Analysis of bars an beams: Theoretical Formulations, Journal of Sound and Vibration, 192, 281-305, 1996.

[16] P. Hardy, L. Jezequel and M. N. Ichchou, Absorption coefficient and energy flow path identification by means of inverse local energy method, The Journal of the Acoustical Society of America, 2320-2320, 112, 5, 2002.

[17] M. N. Ichchou and L. Jezequel, Comments on simple models of the energy flow in vibrating membranes and transversely vibrating plates, Journal of Sound and Vibration, 195, 679-685, 1996.

[18] H. Kuttruff, Room acoustics, Elsevier, 3, 1991.

[19] H. Kuttruff, Energetic Sound Propagation in Rooms, Acoustica with Acta Acoustica, 622-628, 83, 1997.

[20] S. Besset, M. Ichchou and L. Jézéquel, A coupled BEM and energy flow method for mid-high frequency internal acoustic, Journal of Computational acoustic, 2009 , in press.

[21] R. Ohayon and C. Soize, Structural Acoustics and Vibration: Mechanical Models, Variational Formulations and Discretization, Academic Press, 1997. 


\section{List of Figures}

$1 \quad$ Considered system $\ldots \ldots \ldots \ldots \ldots \ldots$

2 element/element interaction . . . . . . . . . . . . 6

$3 \quad$ Numerical simulation methodology $\ldots \ldots \ldots$. . . . . . . . . 9

$4 \quad$ MES/IMES simulation for the plate . . . . . . . . . . . 10

$5 \quad$ MES/IMES simulations for $m=4(\xi=0.06)$ (a), $m=9(\xi=0.14)$ (b), $m=32(\xi=0.5)$ (c) and $m=64(\xi=$

$6 \quad$ FEM/IMES simulations for $\xi=0.06(\mathrm{a}), \xi=0.14$ (b), $\xi=0.5$ (c) and $\xi=1$ (d) 17

$7 \quad$ FEM/IMES simulations for $f_{c}=800 \mathrm{~Hz}$ (a), $f_{c}=900 \mathrm{~Hz}$ (b), $f_{c}=950 \mathrm{~Hz}$ (c) and $f_{c}=1500 \mathrm{~Hz}$ (d) 18

$8 \quad$ location sensitivity to the variation of center frequency $\ldots \ldots . .19$

$9 \quad$ FEM/IMES simulations for $\eta=0.001$ (a), $\eta=0.01$ (b), $\eta=0.1$ (c) and $\eta=0.2$ (d) 20

$10 \quad$ FEM/SEA simulations for $\eta=0.001$ (a), $\eta=0.01$ (b), $\eta=0.1$ (c) and $\eta=0.2$ (d) 21

11 location (a) and quantification (b) sensitivities to the variation of damping for FEM / IMES and FEM / SEA 2

12 FEM/IMES simulations and sensors repartition for $m=55((\mathrm{a})$ and (b)) and $m=39($ (c) and (d) $) 23$

13 location sensitivity to the variation of $\mathrm{f} \ldots \ldots \ldots . \ldots 24$ 


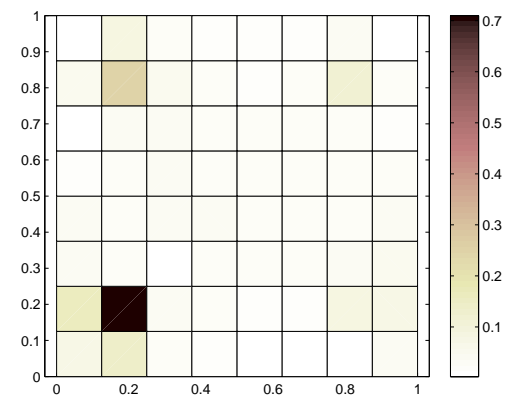

(a)

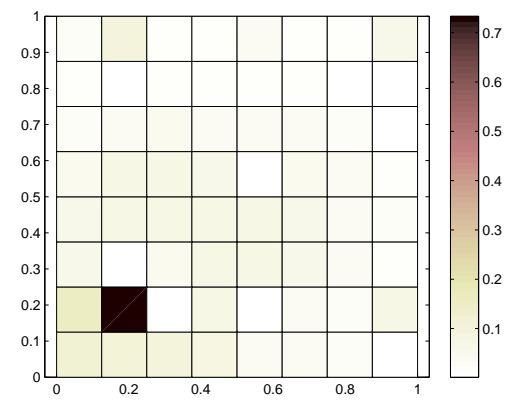

(b)

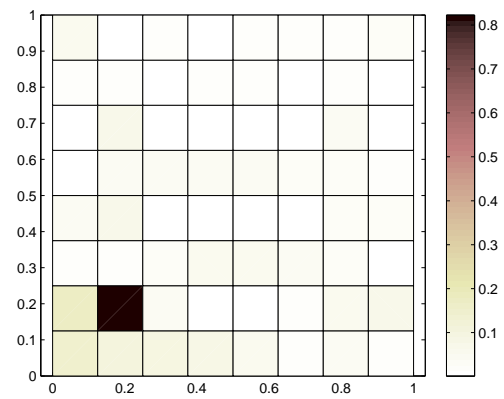

(c)

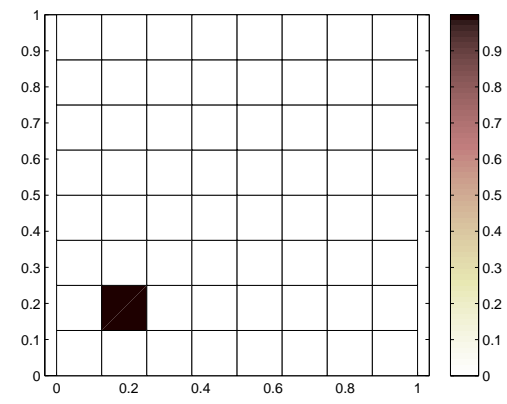

16

(d)

Figure 5: MES/IMES simulations for $m=4(\xi=0.06)$ (a), $m=9(\xi=0.14)$ (b), $m=32(\xi=0.5)(\mathrm{c})$ and $m=64(\xi=1)(\mathrm{d})$ 


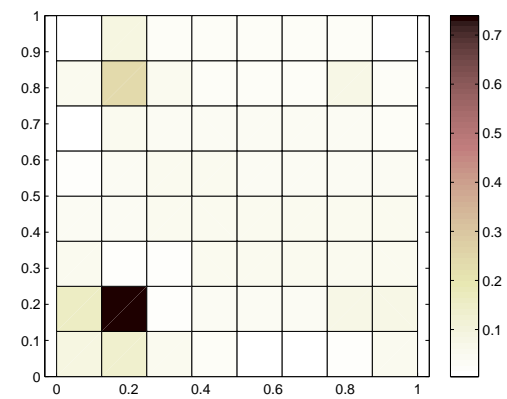

(a)

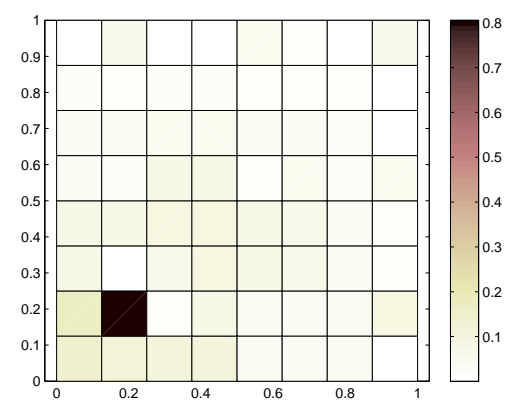

(b)

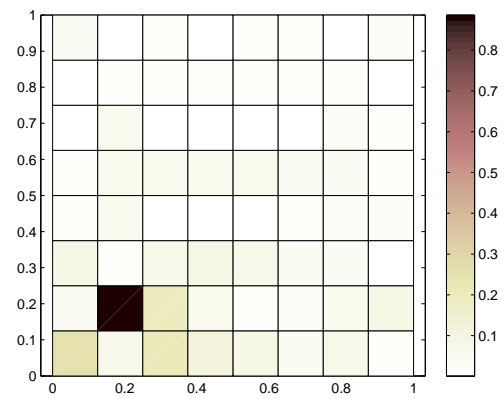

(c)

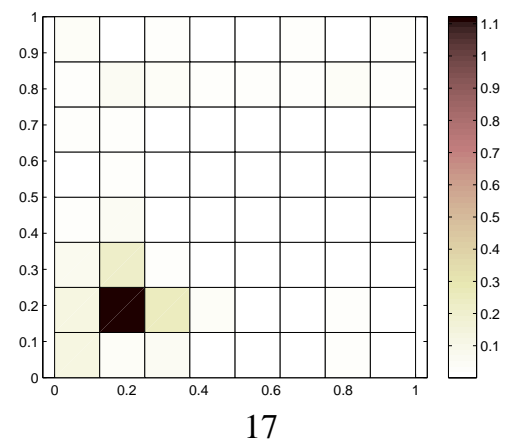

(d)

Figure 6: FEM/IMES simulations for $\xi=0.06$ (a), $\xi=0.14$ (b), $\xi=0.5$ (c) and $\xi=1$ (d) 


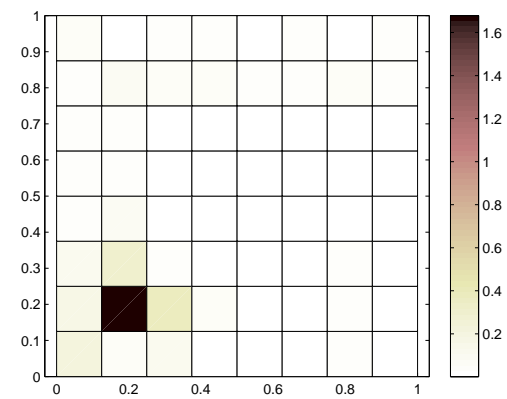

(a)

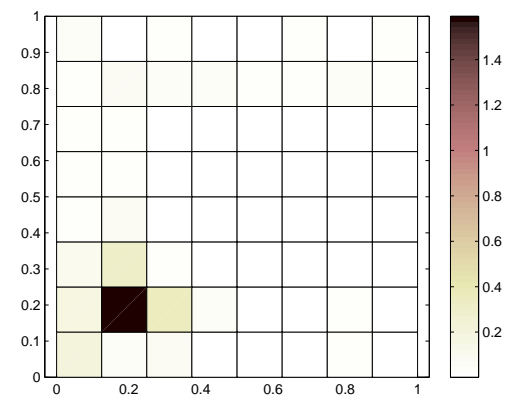

(b)

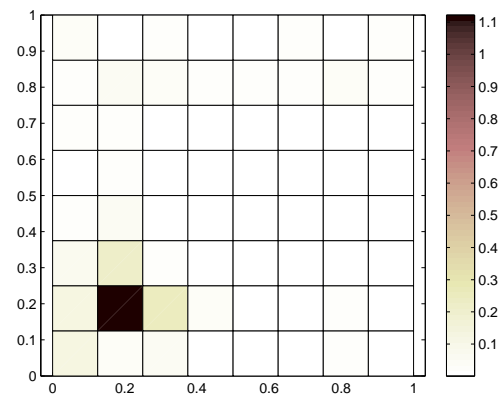

(c)

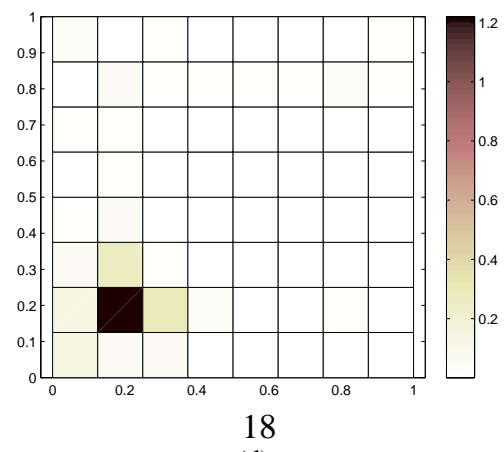

(d)

Figure 7: FEM/IMES simulations for $f_{c}=800 \mathrm{~Hz}(\mathrm{a}), f_{c}=900 \mathrm{~Hz}(\mathrm{~b}), f_{c}=950 \mathrm{~Hz}$ (c) and $f_{c}=1500 \mathrm{~Hz}(\mathrm{~d})$ 


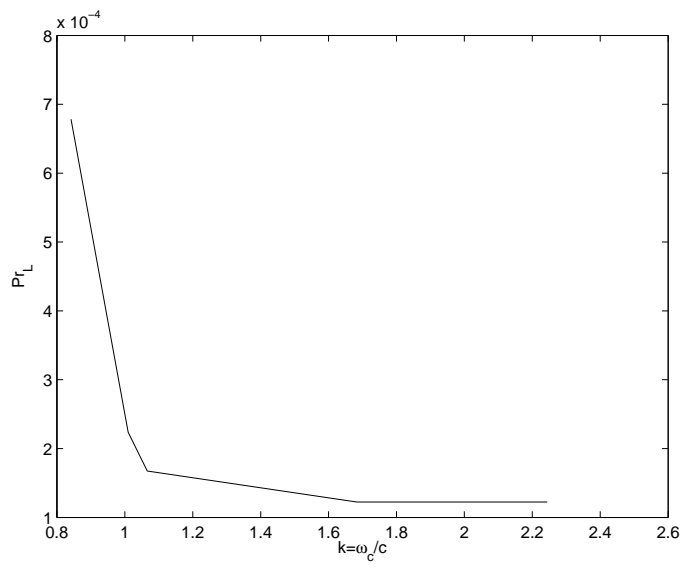

Figure 8: location sensitivity to the variation of center frequency 


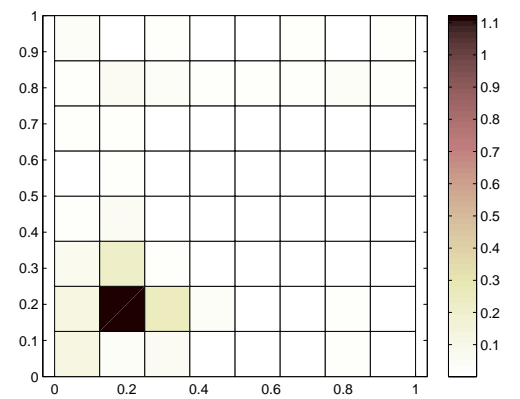

(a)

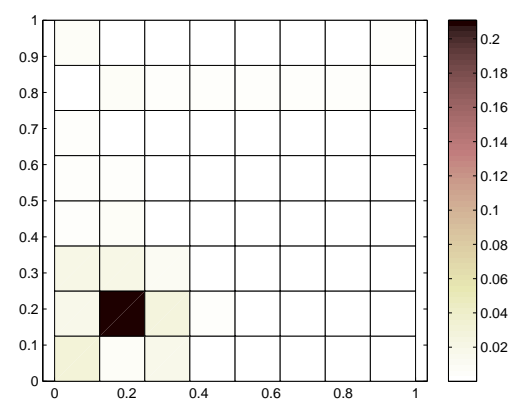

(b)

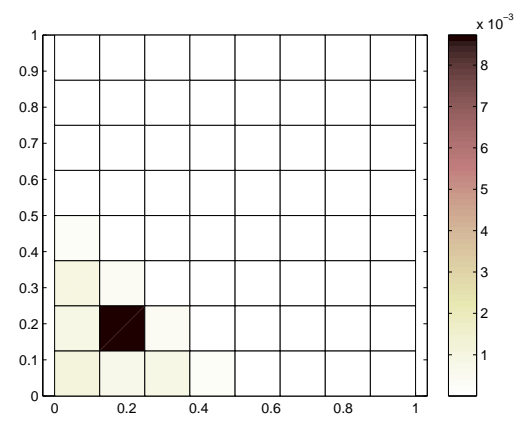

(c)

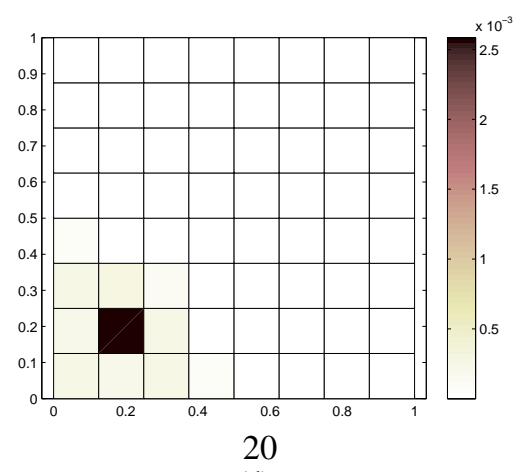

(d)

Figure 9: FEM/IMES simulations for $\eta=0.001$ (a), $\eta=0.01$ (b), $\eta=0.1$ (c) and $\eta=0.2(\mathrm{~d})$ 


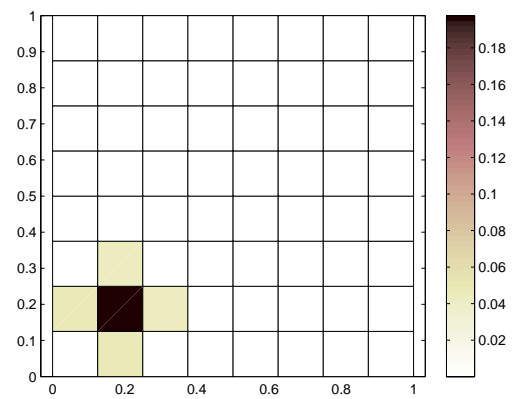

(a)

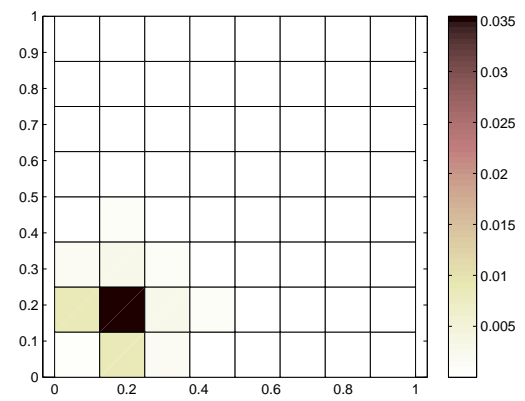

(b)

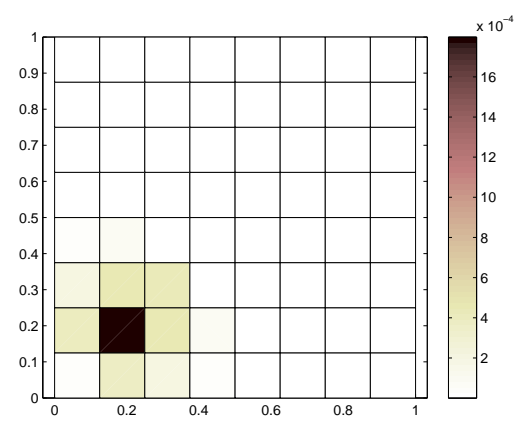

(c)

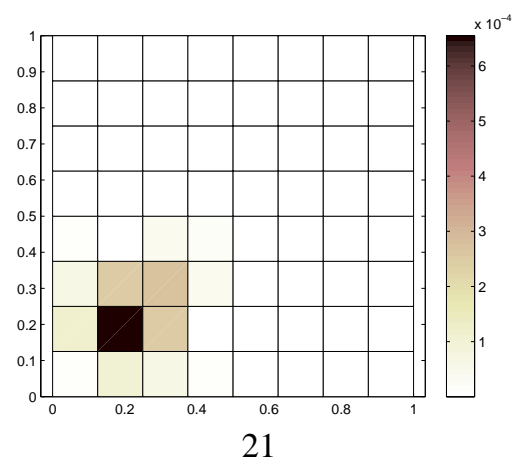

(d)

Figure 10: FEM/SEA simulations for $\eta=0.001$ (a), $\eta=0.01$ (b), $\eta=0.1$ (c) and $\eta=0.2(\mathrm{~d})$ 


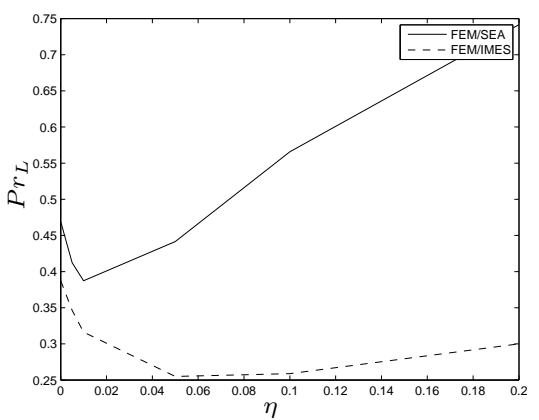

(a)

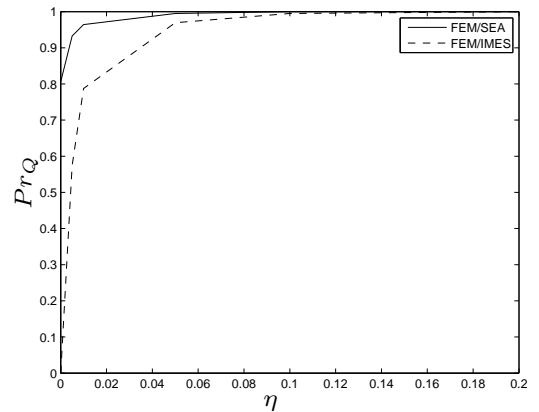

(b)

Figure 11: location (a) and quantification (b) sensitivities to the variation of damping for FEM / IMES and FEM / SEA 


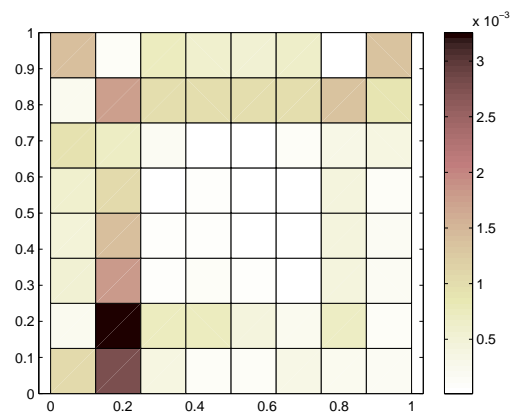

(a)

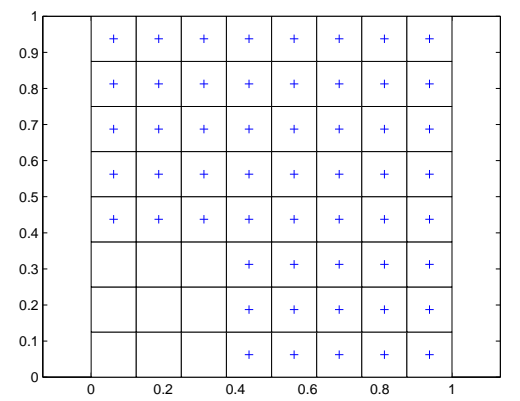

(b)

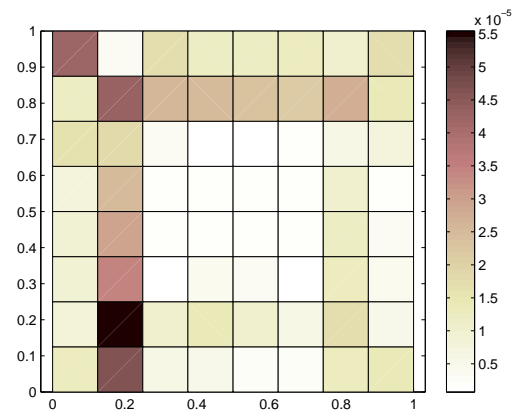

(c)

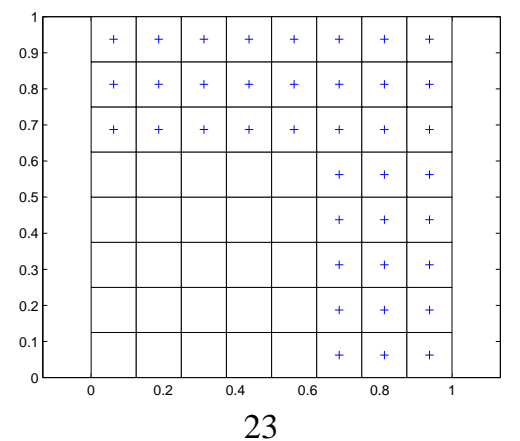

(d)

Figure 12: FEM/IMES simulations and sensors repartition for $m=55$ ((a) and (b)) and $m=39((\mathrm{c})$ and $(\mathrm{d}))$ 


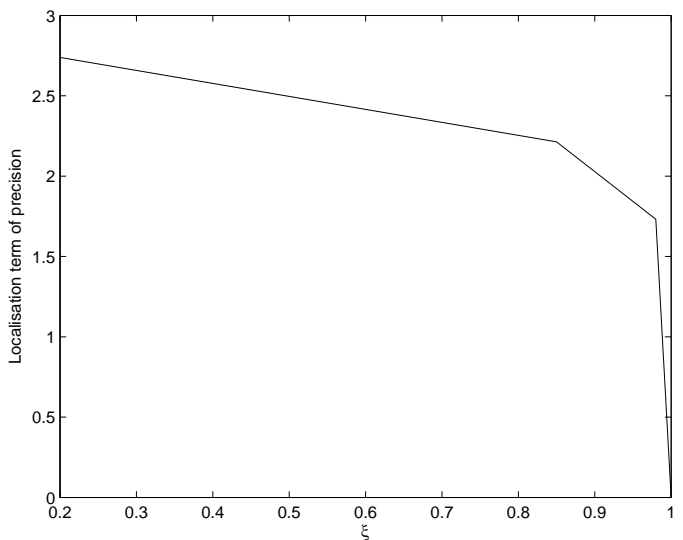

Figure 13: location sensitivity to the variation of $\xi$ 